\title{
Exame preventivo: Conhecimentos e práticas sobre a perspectiva de adolescentes um relato de experiência
}

Preventive examination: Knowledge and practices about the perspective of adolescents an experience report

Examen preventivo: Conocimientos y prácticas sobre la perspectiva de los adolescentes: un informe de experiencia

\section{Resumo}

Descrever a experiência vivenciada de acadêmicas de enfermagem frente a uma ação educativa realizada durante uma extensão universitária. Este estudo corresponde a um relato de experiência do tipo descritivo de abordagem qualitativa, baseado nas vivencias experienciadas durante uma atividade educativa para adolescentes realizadas por grupo de acadêmicas de enfermagem participantes do Projeto de Extensão Educação em Saúde - PROES de um centro universitário privado localizado em Maceió, Alagoas no ano de 2020. A ação ocorreu em uma escola situada no bairro da Cidade Universitária e foi direcionada a estudantes do $1^{\circ}$ ao $3^{\circ}$ ano do ensino médio, com faixa etária entre 14 a 19 anos. A temática abordada foi sobre IST's e exame citopatológico. A realização dessa ação possibilitou o compartilhamento de informações sobre assuntos pertinentes com a vida sexual e reprodutiva, além da oferta de orientações sobre a promoção da saúde e o autocuidado. Os estudantes se mostraram interessados e participativos frente a atividade desenvolvida. Nota-se a importância da realização dessas atividades uma vez que permitem o rastreamento e prevenção de infecções predominantes nesta fase da vida, além de orientações quanto autocuidado, com isso, vale destacar a relevância de atividades voltadas para adolescentes durante o período escolar, mostrando-as a verdadeira necessidade da assistência de enfermagem neste espaço.

Palavras-chave: Educação em saúde; Adolescentes; Citologia; Promoção da saúde em ambiente escolar.

\begin{abstract}
Describe the lived experience of nursing students in front of an educational action carried out during a university extension. This study corresponds to an experience report of a descriptive type with a qualitative approach, based on the experiences experienced during an educational activity for adolescents carried out by a group of nursing students participating in the Health Education Extension Project - PROES of a private university center located in Maceió, Alagoas in the year 2020. The action took place at a school located in the Cidade Universitária neighborhood and was aimed at students from the 1st to the 3rd year of high school, aged between 14 and 19 years old. The theme approached was about STI's and Pap smear. Carrying out this action made it possible to share information on issues relevant to
\end{abstract}


sexual and reproductive life, in addition to offering guidance on health promotion and self-care. Students were interested and participative in the activity developed. The importance of carrying out these activities is noted, as they allow the tracking and prevention of infections prevalent in this stage of life, as well as guidance on self-care, thus, it is worth highlighting the relevance of activities aimed at adolescents during the school period, showing as the real need for nursing care in this space.

Keywords: Health education; Teenagers; Cytology; Health promotion in the school environment.

\section{Resumen}

Describir la experiencia vivida por estudiantes de enfermería frente a una acción educativa realizada durante una extensión universitaria. Este estudio corresponde a un relato de experiencia de tipo descriptivo con enfoque cualitativo, basado en las vivencias vividas durante una actividad educativa para adolescentes realizada por un grupo de estudiantes de enfermería participantes en el Proyecto de Extensión de Educación en Salud - PROES de un centro universitario privado ubicado en Maceió, Alagoas en el año 2020. La acción tuvo lugar en una escuela ubicada en el barrio Cidade Universitária y estaba dirigida a estudiantes de $1^{\circ}$ a $3^{\circ}$ de Bachillerato, con edades comprendidas entre los 14 y los 19 años. El tema abordado fue sobre ITS y examen citopatológico. Esta acción permitió compartir información sobre temas relevantes para la vida sexual y reproductiva, además de ofrecer orientaciones sobre promoción de la salud y autocuidado. Los alumnos mostraron interés y participación en la actividad desarrollada. Se destaca la importancia de realizar estas actividades, ya que permiten el seguimiento y prevención de infecciones prevalentes en esta etapa de la vida, así como la orientación en el autocuidado, por ello, cabe destacar la relevancia de las actividades dirigidas a los adolescentes durante el período escolar, mostrándose como la necesidad real de cuidados de enfermería en este espacio.

Palabras clave: Educacíon para lá salud; Adolescentes; Citología; Promoción de la salud em el entorno escolar.

\section{Introdução}

Este estudo tem como objeto a promoção a saúde para adolescentes no âmbito escolar, voltada para educação sexual e reprodutiva enfatizando a importância da realização do exame preventivo e a prevenção de Infecção sexualmente transmissível (IST’s) como também a estimulação do autocuidado. A motivação para realizar este estudo se deu a partir da percepção do déficit de informações fidedignas acerca da temática abordada, bem como a implementação de estratégias visando uma prática sexual segura.

O exame preventivo citopatológico apresenta-se como um método de rastreamento do câncer de colo do útero (CCU), que contempla mulheres de 25 a 64 anos. Esse exame é realizado com o intuito de detectar lesões precursoras no estágio inicial da doença, ou seja, quando ainda está assintomática, diminuindo assim a morbimortalidade e aumentando as chances de cura. Quando o exame não é realizado periodicamente dificulta a investigação e detecção do câncer de colo de útero assim como de outras patologias (Silveira et al.,2016).

Com as altas taxas de incidência de mulheres que apresentam lesões precursoras na faixa etária de 20 a 29 anos, ressaltase a importância de incluir nos programas de rastreamento adolescentes que tenham vida sexual ativa, visto que indícios patológicos podem ser encontrados de 10 a 15 anos antes da detecção do câncer invasor. A prática de atividade sexual precoce é cada vez mais comum e corriqueira entre os adolescentes. Contudo, essa prática pode provocar um aumento no número de casos de infecção pelo Papilomavírus Humano (HPV) e as IST's (Infecções Sexualmente Transmissível) como abordam Baggio (et al., 2018) e Brasil (2020).

Pesquisas mostram que a contaminação pelo HPV (papiloma vírus humano), acontece no início da vida sexual na adolescência ou em média dos 20 anos de idade, quando a mudança de parceiros é bastante frequente. Outro fator predominante é a não adesão aos métodos contraceptivos de barreira ou uso incorreto de preservativos masculinos e femininos. Em contrapartida, a falta de conhecimentos prévios da doença ou até mesmo o desconhecimento, interferem diretamente no aumento considerável dos riscos para a contaminação pelo vírus (Cavalcante, et al., 2016; Silva, et al., 2015).

Com isso, é importante ressaltar que a educação em saúde para as adolescentes que estão dando início a vida sexual é uma estratégia indispensável, no sentido de aquisição de conhecimento com práticas saudáveis para manutenção, promoção da saúde sexual e reprodutiva, pois é através de ações educativas com orientações em saúde que serão adotadas medidas de cuidados preventivos, ressaltando assim a adesão periódica ao exame ginecológico que é de extrema relevância para o público feminino, 
contudo, através da sua realização que se pode constatar a ocorrência de alguma alteração (Silveira, et al., 2016; Brasil, 2018)

Em 2014, foi inserido no calendário vacinal de meninas de 9 a 14 anos e meninos de 11 a 14 anos, a vacina quadrivalente do Papilomavírus humano (HPV), com a objetivo de prevenir lesões e cânceres futuros que são causados pelo quatro tipo de vírus $(6,11,16$ e 18). A vacina é ofertada gratuitamente pelo Ministério da Saúde (MS) para essa população, pois é através da imunização que os índices de câncer irão diminuir. Estudos apontam que cerca de $70 \%$ a $80 \%$ das pessoas que têm vida sexual ativa irão em algum momento ter contato com o vírus (Brasil, 2020; Interaminense, Oliveira, Leal, Linhares \& Pontes, 2016)

Pesquisas realizadas no Brasil trazem dados importantes sobre o baixo nível de conhecimento dos adolescentes sobre o atendimento em saúde sexual e reprodutiva, esses dados transcorrem diferenças visíveis quando comparado aos outros assuntos sobre sexualidade. Demonstrando que há primordialidade de conduzir ações em programas estudantis, voltados para esses jovens para que ocorra sensibilização da prática do sexo seguro e posteriormente o acompanhamento com um profissional da saúde para realização dos exames preventivos (Silva et al., 2020).

Observa-se que atualmente a prática do sexo tem sido de grande disseminação na adolescência, sedo considerado um problema de saúde pública, todavia, é através da educação em saúde que esse ato pode ser considerado seguro trazendo um bemestar para esses jovens (Interaminense et al., 2016), Diante o exposto, surge o seguinte questionamento: "Quais os conhecimentos e práticas de adolescentes frente ao exame citopatológico? déficit de conhecimento entre a população mais jovem sobre a importância da realização das medidas preventivas incluindo o exame preventivo - uma medida essencial para rastreamento e diagnóstico de doenças prevalentes a partir de práticas e comportamentos inadequados (Silva, Vieira Ferreira, Amaral-Bastos, Monteiro \& Couto, 2020)

Assim, ressalta-se a importância das ações sobre promoção em saúde com o objetivo de levar informações verdadeiras e fundamentais para orientar e sensibilizar os jovens não só sobre as IST's, principalmente sobre a importância dos meios de prevenção e a realização dos exames preventivos, a exemplo da coleta Citopatológica ou popularmente conhecido como citologia. Esse estudo tem como objetivo: relatar a experiência de estudantes durante a aplicação de uma ação educativa sobre exame citopatológico em adolescentes.

\section{Metodologia}

Trata-se de um estudo descritivo, tipo relato de experiência de abordagem qualitativa. Um relato de experiência tratase de um estudo que irá descrever pontualmente a experiência vivenciada, com o intuito de contribuir de modo relevante para uma determinada área estudada. É necessário durante a sua produção desenvolver uma linguagem clara e com riqueza de detalhes para a melhor compreensão do leitor (Dalton \& Farias, 2021).

O presente estudo emerge das atividades desenvolvidas pelo PROES (Projeto de Extensão Educação em Saúde), desenvolvido por um grupo de 6 extensionistas do curso de bacharelado em enfermagem e 2 enfermeiras/professoras do Centro Universitário Mário Pontes Jucá - UMJ, no período de junho de 2019 a junho de 2020. O projeto foi criado com o objetivo de promover ações educativas em saúde e prestar mais assistência para as populações mais vulneráveis. As atividades que foram desenvolvidas a partir da criação do projeto decorrem de uma perspectiva de ofertar informações importantes para melhoria da saúde e sensibilização.

O projeto teve a duração doze meses e como forma organizacional foi criado um cronograma de todas as ações que seriam realizadas. Nesse cronograma eram descritas detalhadamente como as atividades seriam realizadas, o público-alvo, o local e a temática que seria escolhida dependendo da necessidade encontrada.

O planejamento das atividades era discutido através de reuniões mensais, os temas das ações que seriam realizados eram colocados em pauta, a depender do público que seria destinada a ação, as extensionistas faziam a elaboração de slides e cartazes como forma didática, e criação de dinâmicas de forma lúdica para facilitar a compreensão das ouvintes. 
A ação educativa sobre IST's e exame citopatológico foi direcionada a estudantes do $1^{\circ}$ ao $3^{\circ}$ ano do ensino médio, com faixa etária entre 14 a 19 anos, em uma escola situada no bairro da Cidade Universitária - Maceió - AL. Vale destacar a relevância de atividades voltadas para adolescentes durante o período escolar, mostrando-as a verdadeira necessidade do autocuidado com a saúde sexual e reprodutiva.

Elaborou-se um plano de ação para ser realizado no dia em questão. Com isso, foi montado um slide e cartazes como forma didática para apresentação da temática abordada contendo imagens ilustrativas com o objetivo de melhorar o entendimento e assim alcançar a sensibilização dos riscos da adesão à prevenção em saúde.

A ação foi dividida em 3 momentos: no primeiro momento ocorreu o acolhimento e quebra gelo com as participantes, no segundo momento: a apresentação das extensionistas e exposição do objetivo em questão. A justificativa para a temática escolhida foi que as adolescentes tinham dificuldades em procurar o serviço de saúde após a coitarca.

E no terceiro momento se desenvolveu uma roda de conversa sobre a importância da realização do exame citopatológico após a coitarca e algumas IST's. Momento em que houve grande participação das participantes com as extensionistas. Foram indagados vários questionamentos sobre a temática, pois muitas das participantes desconheciam o assunto proposto.

\section{Resultados e Discussão}

Os projetos de extensão desenvolvidos no ensino superior têm um papel primordial na construção de vínculo entre os discentes e a sociedade, visto que a formação ética, humanizada e integral é cada vez mais necessária para essa rede de aproximação. Dessa forma, ocorrerá a criação de novos conhecimentos e aperfeiçoamento de práticas educativas, visando realizar atividades que irão beneficiar toda a comunidade (Freitas, et al 2021; Silva, et al., 2021).

Ao decorrer do desenvolvimento das práticas educativas de extensão os acadêmicos irão ser beneficiados cotidianamente de um amplo aprendizado prático/teórico e assim aperfeiçoando suas habilidades. É através dessas vivências que os mesmos vão desfrutar de experiências únicas e enriquecedoras tanto sob o ponto de vista do ensino durante a graduação como também para a vida profissional (Ferreira, Suriano \& Domenico 2018)

Sabe-se a importância do desenvolvimento das práticas de educação em saúde direcionadas a adolescentes, onde essas atividades mostram grande eficiência quando aplicadas de forma educativo/preventiva ainda no contexto escolar, proporcionando assim o incentivo a adesão do autocuidado através do protagonismo e autonomia dessas jovens em relação a sua saúde, com o intuito da diminuição da exposição a situação de risco (Silva et al., 2020).

Sabe-se que o início da vida sexual ativa tem acontecido entre os jovens mais cedo nas últimas décadas, devido a diversos fatores que atualmente tem modificado os costumes sociais e as interpretações sobre o ato sexual, que em tempos passados isso era considerado um "tabu" ou "um assunto proibido" para conversar com adolescentes, entretanto atualmente por meio do acesso a tecnologias a disseminação de informações que a mídia oferta e as diversificadas redes sociais vem quebrando certas concepções e modificando os comportamentos humanos (Silva, et al., 2020; Gonçalves, et al., 2015).

Segundo o levantamento do UNICEF (Unidas para Fundo Internacional de Emergência das Nações a Infância) embora que em 2019 houve uma diminuição nos números de adolescentes grávidas em Alagoas, cerca de 25\% dos bebês que nascem no estado entre 2016 -2019, são de mães adolescentes, onde a faixa etária está entre 15 a 19 anos, esse mesmo levantamento mostra que cerca de 1,58 \% nascem de mães com idade entre 10 a 14 anos. A gravidez neste grupo populacional vem sendo considerada um grande problema de saúde pública, uma vez que pode acarretar complicações obstétricas para com a mãe e o recém-nascido.

Diante disso, nota-se a importância da utilização de estratégias práticas para o público em questão, visto que este apresenta a necessidade de orientações sobre atividades educativas em saúde, visando a realização do exame citopatológico e a estimulação do autocuidado, destacando o uso correto do preservativo como principal forma de prevenção contra as IST's. Nisso, segundo a SBP (Sociedade Brasileira de Pediatria), a importância de orientações sobre os riscos de IST e gravidez não planejada, 
apontando as atividades sexuais como fonte de prazer e sendo inseridas no processo do desenvolvimento do adolesceste, deve ser priorizada. Tal conduta deve ser livre de julgamentos morais, de preconceitos e estereótipos, possibilitando ao adolescente perceber seu profissional de saúde como um interlocutor aberto para as dúvidas na área da sexualidade.

Diante disso, durante a ação efetivada pelo projeto para o grupo de adolescentes, uma das principais estratégias utilizadas foi a criação de um vínculo através da roda de conversa, em que as extensionistas verificaram o nível de conhecimento por meio de perguntas relacionadas ao assunto, dentre elas se sabiam algo sobre o exame?, como era realizado?, quando fazer?, como era realizado?, se alguma das presentes já tinha realizado?, se elas faziam uso de algum método contraceptivo?, se tinham alguma experiência que gostariam de dividir?.

Nesse momento foi observado que um número reduzido de alunas que estavam presentes, responderam que já tinham realizado o exame. Não obstante, em uma quantidade limitada, relataram que sabiam algo sobre o assunto, e contrapartida boa parte das participantes relataram que não sabiam quase nada ou nada do exame citopatológico. Nesta perspectiva, de acordo com Silva (et al., 2020) o déficit de informação neste público refere a falta de diálogo com profissionais capacitados, assim como a falta da implementação dos programas existentes para este público no espaço escolar.

Pesquisas realizadas no Brasil trazem dados importantes sobre o baixo nível de conhecimento dos adolescentes sobre o atendimento em saúde sexual e reprodutiva, esses dados transcorrem diferenças visíveis quando comparado aos outros assuntos sobre sexualidade (Silva, et al., 2020). Demonstrando que há primordialidade de conduzir ações em programas estudantis, voltados para esses jovens para que ocorra sensibilização da prática do sexo seguro e posteriormente o acompanhamento com um profissional da saúde para realização dos exames preventivos (Almeida, et al., 2017).

Dando continuidade à atividade, as acadêmicas de enfermagem explanaram sobre os principais sinais e sintomas, diagnóstico, forma de transmissão e tratamento das IST's utilizando-se de imagens ilustrativas das infecções que mais acometem esse público, em especial, HPV, sífilis e herpes. Diante desse cenário, as alunas se mostraram muito surpresas com as imagens que foram expostas e participativas, fazendo questionamentos sobre as infecções, em destaque para a forma de contágio, o risco sobre o ato sexual desprotegido, o desconhecimento e a não utilização de métodos preventivos.

Com base nisso, foi ressaltado a importância do uso correto de métodos de barreira, como os preservativos masculino e feminino, como forma de prevenção de várias patologias. Durante esse momento de orientações ocorreu a demonstração do uso correto de preservativo masculino através de uma prótese de genitália feminina e masculina. As extensionistas solicitaram a participação das estudantes para demonstrarem o uso correto do preservativo. Lara (et al., 2016) relata que o uso de abordagens lúdicas que possam chamar a atenção do adolescente representa uma importante estratégia de adesão ao conhecimento ao mesmo tempo que possibilita a sensibilização para prática de vida.

Nota-se que a falta de acesso às informações básicas sobre o assunto é uma das maiores causas da não adesão ao exame preventivo, é devido a essa ausência de conhecimento sobre os benéficos da sua realização que pode ocorrer também o agravamento dos principais problemas de saúde, levando ao adoecimento precoce desses jovens. Pode-se ressaltar que muitas das vezes o acesso a essas informações é através de canais de comunicação como a internet, que podem apresentar um risco se não utilizados com cautela, devendo sempre procurar sempre fontes confiáveis (Viero et al., 2015).

No decorrer dessa ação foi evidenciando a importância da realização do exame preventivo entre as adolescentes após a coitarca. No decurso do bate-papo deixou-se claro que as adolescentes poderiam realizar questionamentos ou contar algum fato que elas experienciaram, possibilitando a troca de conhecimento e histórias entre as participantes, uma vez que algumas compartilharam suas vivências.

Outrossim, sabe-se que a realização do exame é considerada uma medida eficaz no rastreamento e prevenção de doenças. A orientação é uma estratégia primordial para o esclarecimento sobre os riscos que essas jovens estão suscetíveis, o maior desafio da promoção em saúde é a adesão a esse exame que é essencial para a saúde sexual das adolescentes (Silva et al., 
2016).

Esse exame deve ser feito por profissionais da área da saúde devidamente capacitados e habilitados, por meio de um procedimento realizado de forma rápida e indolor, podendo causar apenas um pequeno incômodo em algumas mulheres, e outras não apresentam nenhuma queixa, realizando assim o acolhimento com escuta qualificada para essa usuária. Espera-se que a coleta realizada através desse exame seja executada na técnica correta para obtenção de uma amostra satisfatória do esfregaço cervical (Brasil, 2016).

Durante a ação, foi explicitado também, a temática sobre o câncer de mama, reproduzindo uma simulação com mamas feitas de pano pelas extensionistas, mostrando-as de como deveria ser realizado o toque de maneira correta. Advertindo-as sobre a aparição de tumores benignos e palpáveis que podem não evoluir sendo identificados e tratados, e nada substituiria a consulta com a ginecologista ou qualquer outro profissional de saúde para a retirada de dúvidas, e que, a detecção precoce do câncer de mama por meio do diagnóstico precoce: exame mamografia ou ultrassonografia, tem o um único objetivo de identificar a doença em fase inicial, e tratá-lo, pois, as chances de cura na fase inicial são altas.

Nos últimos anos, o Instituto Nacional de Câncer (INCA) tem trabalhado com a população feminina a importância de "estar alerta" a qualquer alteração suspeita nas mamas (estratégia de conscientização), assim como tem desenvolvido ações com gestores e profissionais de saúde sobre a importância do rápido encaminhamento para a investigação diagnóstica de casos suspeitos e início do tratamento adequado, quando confirmado o diagnóstico (Brasil, 2020).

Ao final, de todo conteúdo exposto realizou-se um jogo lúdico como método de aprendizagem em forma de "quiz", o mesmo foi realizado objetivando a estimulação dos conhecimentos aprendidos por elas no decorrer das atividades, dando ênfase aos assuntos abordados durante a ação, sanando possíveis dúvidas, sensibilizando quanto a importância das temáticas e primordialmente o autocuidado nessa fase de vida.

Em vista da realização dessa atividade com grupo de extensionistas para com as adolescentes permitiu constatar a importância da formação e condução de grupos de educação em saúde nas escolas e que há sim, a necessidade de realizar de forma sistemática um material educativo para promoção e prevenção da saúde destes adolescentes. Contudo, ressalta a autonomia do acadêmico de enfermagem frente às práticas educativas no contexto real, em especial para adolescentes dentro do espaço escolar, haja visto as contribuições tanto para os graduandos assim como para o público em questão.

\section{Conclusão}

Diante do exposto observa-se a relevância de práticas educativas para o público de adolescentes no espaço escolar frente a sensibilização de temas pertinentes ao autocuidado e a saúde sexual e reprodutiva. Ademais, ressalta que as contribuições das atividades de extensão favorecem o aprimoramento de habilidades, assim como a atuação do graduando no cenário real, ao mesmo tempo possibilita benefícios para população, neste caso para grupo de jovens no qual foi implementada a ação aqui ressaltada.

Em suma, observa-se ainda que esta prática proporcionou aos acadêmicos de enfermagem uma experiência construtiva, satisfatória e viável, embora considere-se que muito há a ser conhecido e praticado na área, principalmente para os adolescentes no ambiente escolar. Outrossim, a experiência vivenciada impulsiona os extensionistas a ampliar seus conhecimentos com base científicas, ao mesmo tempo aperfeiçoando suas habilidades teórico-prático.

Em contrapartida, notou-se a necessidade de realizar mais ações voltadas para estas temáticas com adolescentes, já que se visualiza o déficit de conhecimento nesse espaço, além dos tabus que circundam o autocuidado e a saúde sexual e reprodutiva de jovens. Sendo fundamental a implementação de medidas e estratégias de promoção à saúde e orientações frente à prevenção de doenças que mais acometem esse público.

Nessa perspectiva, percebeu-se a restringência de estudos voltados a realização do exame citológico para o grupo em 
questão, com isso ressalta a importância da execução de práticas educativas embasada cientificamente como forma de elaboração e publicação de pesquisas. Diante do exposto, sugere-se o desenvolvimento de atividades educativas em saúde vinculada ao conhecimento teórico prático impulsionando a construção para publicações futuras.

\section{Referências}

Almeida, R. A. A. S, Corrêa, R. da G. C. F, Rolim, I. L. T. P, Hora, J. M. da, Linard, A. G, Coutinho, N. P. Silva, \& Oliveira, P. da S. (2017). Conhecimento de adolescentes relacionados às doenças sexualmente transmissíveis e gravidez. Revista Brasileira de Enfermagem, 70(5), 1033-1039. https://doi.org/10.1590/00347167-2016-0531

Baggio K, Silva B, Prestes M. O, Diehl C, Ferraz M, Coser J, \& Zanella, J. P. (2018). Exame de papanicolaou em adolescentes e mulheres jovens: análise do perfil citológico. Adolesc Saude. 15(3):44-51 http://adolescenciaesaude.com/detalhe_artigo.asp?id=732\#

Brasil. (2020). Ministério da Saúde do Brasil. Infecções sexualmente transmissíveis- IST. https://antigo.saude.gov.br/saude-de-a-z/infeccoes-sexualmentetransmissiveis-ist

Brasil. (2020). Ministério da saúde do Brasil. Instituto Nacional de câncer- INCA. Outubro Rosa. Https://www.inca.gov.br/campanhas/cancer-demama/2020/outubro-rosa-2020

Brasil. (2016). Ministério da Saúde do Brasil. Protocolos da Atenção Básica: Saúde das Mulheres / Ministério da Saúde, Instituto Sírio-Libanês de Ensino e Pesquisa. http://bvsms.saude.gov.br/bvs/publicacoes/protocolos_atencao_basica_saude_mulheres.pdf. Acesso em $28 \mathrm{mar} 2020$.

Brasil. (2016). Instituto Nacional de Câncer José Alencar Gomes da Silva. Coordenação de Prevenção e Vigilância. Divisão de Detecção Precoce e Apoio à Organização de Rede. Diretrizes brasileiras para o rastreamento do câncer do colo do útero. INCA, http://www.citologiaclinica.org.br/site/pdf/documentos/diretrizes-para-o-rastreamento-do-cancer-do-colo-do-utero

Brasil. (2018). Ministério da Saúde do Brasil. Proteger e cuidar da saúde de adolescentes na atenção básica. http://bvsms.saude.gov.br/bvs/publicacoes/proteger_cuidar_adolescentes_atencao_basica_2ed.pdf

Brasil. (2020). Ministério da Saúde do Brasil. Vacinação HPV. https://portalarquivos.saude.gov.br/campanhas/vacinahpv/

Cavalcanti É. F. F, Silva C. R, Vasconcellos M. M, Silva M. G. G, Torres M. C. M. B, \& Torres SR. (2016). Contribuição ao estudo da infecção pelo HPV em adolescentes: estratégias e desafios na abordagem desse grupo. Adolesc Saude. https://adolescenciaesaude.com/detalhe_artigo.asp?id=595\&idioma=Portugues\#

Daltro, M. R; FARIA, \& A. A de. (2019). Relato de experiência: Uma narrativa científica na pós-modernidade. Estudos e Pesquisas em Psicologia, Rio de Janeiro, (19) https://www.e-publicacoes.uerj.br/index.php/revispsi/article/view/43015/29726.

Silva, A., Sousa, S. C. S., Chaves, A. C. F., Sousa, S. G. da C. Andrade, T. M. de, \& Filho, R. da R. (2019). Importância da Extensão Universitária na Formação Profissional: Projeto Canudos. Revista de Enfermagem UFPE online, 13. https://doi.org/10.5205/1981-8963.2019.242189.

Ferreira, P. B; Suriano, M. L. F, \& Domenico, E. B. L. (2018). Contribuição da Extensão Universitária na formação de graduandos em Enfermagem. Rev. Ciênc. (14)3. https://core.ac.uk/download/pdf/300077182.pdf.

Freitas, T., Paula, C., Zanon, B., Meirelles, F., Welleir, T., \& Padoin, S. (2016). Contribuições da extensão universitária na formação de acadêmicos de enfermagem. Revista de Enfermagem da UFSM, 6(3), 307 - 316. doi: https://doi.org/10.5902/2179769219966

Gonçalves, H., Machado, E. C., Soares, A. L. G, Camargo-Figuera, F. A, Seerig, L. M., Mesenburg, M. A, Guttier, M. C, Barcelos, R. S, Buffarini, R, Assunção, M. C. F, Hallal, P. C, \& Menezes, A. M. B. (2015). Início da vida sexual entre adolescentes (10 a 14 anos) e comportamentos em saúde. Revista Brasileira de Epidemiologia, 18(1), 25-41. https://doi.org/10.1590/1980-5497201500010003

Interaminense, I. N. da C. S., Oliveira, S. C de, Leal, L. P, Linhares, F. M. P., \& Pontes, C. M. (2016). Tecnologias Educativas Para Promoção Da Vacinação Contra O Papilomavírus Humano: Revisão Integrativa Da Literatura. Texto \& Contexto - Enfermagem, 25(2), e2300015.https://doi.org/10.1590/010407072016002300015

Lara, S, Salgueiro, A. C. F, Copetti, J, Lanes, K. G, Puntel, R. L \& Folmer, V. (2016) Educação e saúde no contexto escolar: uma experiencia de abordagem lúdica com o tema saúde cardiovascular nos anos iniciais. (16) 65-82. DOI: https://doi.org/10.22456/2595-4377.31945

Silva, A. de S. N, Silva, B. L. C. N, Silva Júnior, A. F. da, Silva, M. C. F da, Guerreiro, J. F, \& Sousa, A. do S. C. de A. (2015). Início da vida sexual em adolescentes escolares: um estudo transversal sobre comportamento sexual de risco em Abaetetuba, Estado do Pará, Brasil. Revista Pan-Amazônica de Saúde, 6(3), 27-34. http://scielo.iec.gov.br/scielo.php?script=sci_arttext\&pid=S2176-62232015000300004\&lng=pt\&tlng=pt

Silva, L. S. R, Lessa, E. C. Silva, T. M. da, Albuquerque, A. K. D. da S. Ferreira, M. D. R \& Silva, T. L. L da. (2016). Adesão ao Exame Papanicolau por Mulheres Jovens em Unidade Básica de saúde, Revista de Enfermagem, Recife, (10)12, $\mathrm{p}$ 4637-4645. https://pdfs.semanticscholar.org/ffb6/54ba51bcec332a2676920c7756e7f9c0e41c.pdf

Silva, S. M. D. T da, Vieira Ferreira, M. M da S, Amaral-Bastos, M. M, Monteiro, M. A. J, \& Couto, G. R. (2020). Diagnóstico do conhecimento dos adolescentes sobre sexualidade. Acta Paulista de Enfermagem, 33, eAPE20190210.https://doi.org/10.37689/acta-ape/2020ao0210

Silveira, N. S. P, Vasconcelos, C. T. M, Nicolau, A. I. O, Oriá, M. O. B, Pinheiro, P. N. da C, \& Pinheiro, A. K. B. (2016). Conhecimento, atitude e prática sobre o exame colpocitológico e sua relação com a idade feminina. Revista Latino-Americana de Enfermagem, 24, e2699.https://doi.org/10.1590/1518-8345.0700.2699 
Research, Society and Development, v. 10, n. 8, e16810817078, 2021

(CC BY 4.0) | ISSN 2525-3409 | DOI: http://dx.doi.org/10.33448/rsd-v10i8.17078

Viero, V. d. S. F, Farias, J. M de, Ferraz, F, Simões, P. W, Martins, J. A, \& Ceretta, L. B. (2015). Educação em saúde com adolescentes: análise da aquisição de conhecimentos sobre temas de saúde. Escola Anna Nery, 19(3), 484-490. https://doi.org/10.5935/1414-8145.20150064

UNICCEF. Fundo Internacional de Emergência das Nações Unidas para a Infância. (2020). O mapa dos direitos sexuais e dos direitos reprodutivos de adolescentes em Maceió. https://www.unicef.org/brazil/relatorios/plataforma-dos-centros-urbanos-2017-2020/mapa-direitos-sexuais-adolescentes-maceio. 